\title{
LIGHTING AS A CIRCADIAN RHYTHM-ENTRAINING AND ALERTNESS- ENHANCING STIMULUS IN THE SUBMARINE ENVIRONMENT
}

Loring J. Crepeau ${ }^{1}$, John D. Bullough ${ }^{2}$, Mariana G. Figueiro ${ }^{2}$, Steven Porter ${ }^{3}$, and Mark S. Rea ${ }^{2}$

${ }^{1}$ Naval Submarine Medical Research Laboratory, Groton, CT 06349-5900

${ }^{2}$ Lighting Research Center, Rensselaer Polytechnic Institute, 21 Union Street, Troy, NY 12180

${ }^{3}$ General Dynamics Electric Boat Division

\section{Summary}

The human brain can only accommodate a circadian rhythm that closely follows 24 hours. Thus, for a work schedule to meet the brain's hard-wired requirement, it must employ a 24 hour-based program. However, the 6 hours on, 12 hours off (6/12) submarine watchstanding schedule creates an 18- hour "day" that Submariners must follow. Clearly, the 6/12 schedule categorically fails to meet the brain's operational design, and no schedule other than one tuned to the brain's 24 hour rhythm can optimize performance.

Providing Submariners with a 24 hour-based watchstanding schedule - combined with effective circadian entrainment techniques using carefully-timed exposure to light - would allow crewmembers to work at the peak of their daily performance cycle and acquire more restorative sleep.

In the submarine environment, where access to natural light is absent, electric lighting can play an important role in actively entraining - and closely maintaining - circadian regulation. Another area that is likely to have particular importance in the submarine environment is the potential effect of light to help restore or maintain alertness.

\section{Introduction}

Since the $13^{\text {th }}$ century, maritime workers have utilized a 4 hours on, 8 hours off (4/8) watch schedule that continued into the Polaris submarine patrols of the early 1960s. However, because modern Submariners must also train, qualify, and conduct drills when not on watch, the $4 / 8$ schedule prevented them from obtaining sufficient sleep during their off-watch periods. During prolonged patrols, Submariners suffered from progressive sleep debt. To remedy this, the 6 hours on, 12 hours off $(6 / 12)$ schedule was adopted.

In 1969, Stolgitis[1] reported the 6/12 schedule provides Submariners adequate opportunity to obtain sufficient sleep, even with the high demand inherent in the modern submarine milieu. To rationalize employing the 6/12 schedule, Stolgitis calculated Submariners average 8.65 hours of sleep time available every 24 hours. However, this statistic was calculated across 72 hours. Moreover, while the average sleep time appears adequate, the Submariner enjoyed only 5 hours available to sleep during the first 24 hours; the three respective sleep periods available during the following 48 hours were $4.5,6$, and 10.5 hours. Finally, the study critically failed to acknowledge the brain's powerful, 24 hour-based circadian performance rhythm, or how the $6 / 12$ fails to accommodate this rhythm. For example, the periods when the Submariner must 
stand watch and pursue sleep following the 6/12 rotates around the clock, thereby requiring him to work and rest at times in direct conflict with the brain's 24 hour rhythm.

\section{Overview of Circadian Rhythms}

All organisms - humans included - undergo continuous rhythms of various physiological and behavioral functions with an inherent period near 24 hours, entrained by millions of years of exposure to the natural solar day. Such rhythms have been known to exist in humans for several decades[2], and are called circadian rhythms ( $\operatorname{circa}=$ about, die $=$ day)[3]. Circadian rhythms that have been studied in humans and other mammals[4] include: sleep-wake cycles (with sleep normally occurring during the solar night in diurnal species, and during the solar day in nocturnal species); core body temperature rhythms (with the maximum temperature occurring during the solar day and the minimum temperature at night); hormone production rhythms (for example, the hormone melatonin is produced during the solar night, while little to none is produced during the day); and alertness and performance.

Hence, there are differentially advantageous times during the day and night for working (i.e., highest output, lowest errors) and obtaining efficient sleep (i.e., maximally restorative). Following work/rest schedules that fail to accommodate the brain's circadian timing for performance and sleep induces circadian desynchronization. Circadian desynchronization is the culprit that causes people to suffer jet lag, whenever travel abruptly delivers them into a time zone and activity schedule typically displaced by several hours or more.

Circadian desynchronization is also known to induce other adverse conditions, including fatigue, insomnia, diminished concentration, irritability, and exhaustion with mild depression[5-7]. Finally, it can also degrade health, elevating the risk of cardiovascular disease $[8,9]$, perhaps by increasing the likelihood of developing obesity and elevated circulating triglycerides[10], gastrointestinal disease, and reproductive dysfunction[9].

\section{The influence of Light on Circadian Rhythms}

Most circadian rhythms appear to be largely under the influence of the suprachiasmatic nucleus (SCN) in the brain, which receives input about environmental conditions. The SCN transmits this information throughout the body about the time of day, thereby functioning as the master clock of the body. The mammalian SCN's most important environmental input appears to be light and darkness transmitted from the eyes' retinas via the retinohypothalamic tract. Other environmental conditions, including temperature, sound, and availability of meals can also influence the regulation of circadian rhythms, but the light-dark cycle of the solar day exerts the largest impact on circadian regulation[4].

For terrestrial workers, working the night shift proves especially problematic, since daily sunlight exposure at the end of the shift recruits and maintains the same day-night circadian performance cycle in night workers that day workers follow. Night shift workers are thus chronically compelled to work and pursue sleep at times directly contradicting the optimal times in their circadian rhythms. Workers confronted with this temporal opposition demonstrate diminished performance. This negative influence is often compounded, since 
night workers also typically suffer chronic sleep debt, a condition that further reduces alertness and cognitive performance, and elevates the likelihood of committing errors and accidents[11].

Long after the initial discovery of circadian rhythms, it was assumed that human beings had evolved to the point where circadian rhythms were no longer impacted by the light-dark environment. The finding by Lewy et al.[12] that bright white light delivered to humans' eyes at night could inhibit melatonin production - and even shift the melatonin production rhythmdemonstrated that this earlier belief about lighting was false. Since 1980, research has identified many of the near- and longer-term effects of light on circadian-related functioning in humans.

\section{The 6/12 Watchstanding Schedule versus the Brain}

Requiring Submariners to follow the 6/12 schedule is akin to having them instantaneously travel East across six time zones. Once there, they must attempt to accommodate that zone's work/rest pattern before 'taking off' again 18 hours later. By virtue of the schedule they must follow, Submariners are in a nearly constant state of "operational jet lag." Because watchstanders' work environment is bereft of the sun's influence, their circadian rhythms were reported to "free run" on a cycle that averaged 24.35 hours, in spite of 24 hour-based stimuli including social contacts, meals, and knowledge of clock time[13].

Naval Submarine Medical Research Laboratory evaluated a 24 hour based watchstanding schedule during an operational tour aboard the USS MARYLAND, a U.S. Navy ballistic missile submarine. Each watch section was assigned a fixed, 8 hour (FIXED 8) watchstanding shift (i.e., 2400-0800; 0800-1600, and 1600-2400), and prescribed a sacrosanct 8-hour sleep period. Accommodating this new schedule also required coordinating the FIXED 8 schedule with the submarine's operational commitments that included training, drills, maintenance, and cleaning. A post-study questionnaire revealed crewmembers more positively rated the FIXED 8 over the $6 / 12$ on several factors, including fatigue and energy levels, sleep inertia, and crew morale.

Still, to take full advantage of this watchstanding scheduling strategy requires conscious and active circadian entrainment. This is because circadian rhythms, while easily entrained using natural sunlight, can fall into a free-running status when lighting levels remain low. Moreover, the 18 hour "day" that watchstanders follow nullifies 24 hour-based non-light cues that normally help entrain circadian rhythms, including activity and feeding [13]. Active entrainment using new lighting systems would likely be needed to maximize performance during the work shift and minimize activity during rest periods. Lighting would also prove a crucial tool when the watch shifts were rotated among the sections to rapidly re-entrain Submariners to a new shift schedule.

\section{Near-Term Effects of Light}

One reason that electric lighting was thought to have negligible impact on human circadian rhythms is because light levels used to study this effect were historically insufficient. Lewy et al.[12] showed that white light was sufficient to reliably inhibit human nocturnal melatonin production only when the illuminances at subjects' eyes were 2500 lux. In comparison, typical horizontal light levels in buildings are 300 to 500 lux, providing about 60 to 100 lux at 
occupants' eyes[14]. Later studies showed that with careful control, several hundred lux of white light — which is still relatively bright — incident upon subjects' eyes could suppress melatonin[1518]. Similarly, bright light has been shown to cause short-term increases in body temperature, and even to temporarily increase feelings of wakefulness and alertness[19].

\section{Longer-Term Effects of Light}

The free-running period of human circadian rhythms is not exactly 24 hours; in fact it is slightly longer[4]. Thus, the 24 hour solar day acts not only in a near-term way to affect circadian-related functions (e.g., to suppress hormone production or increase body temperature) but, through the $\mathrm{SCN}$, it also directly entrains these functions to a 24 hour day. Depending upon when an individual is exposed to bright light, that light could advance (cause to occur earlier than normal), or delay (cause to occur later than normal) the rhythm of circadian functions. As an example, light exposure during the morning will generally advance the circadian clock, while light exposure during the evening delays it. Following east-west travel, for example, the delayed shift in clock time caused by the solar day at the new location will eventually induce delayed rhythms of temperature, melatonin and other functions. These shifts are caused mainly by the light-dark cycle of the new location, and only secondarily through other cues such as meals.

\section{Characteristics of Light and Lighting}

Fundamentally, the effective application of lighting involves the control of several basic characteristics of light include quantity, spectrum, distribution, timing, and duration. As described above, the characteristics of light that support human vision differ from those impacting the circadian system[20,21]. These characteristics are shown in Table 1 (and described below).

\begin{tabular}{|c|c|c|c|}
\hline \multirow{2}{*}{$\begin{array}{c}\text { Lighting } \\
\text { characteristics } \\
\text { (broad-band } \\
\text { light) }\end{array}$} & \multicolumn{3}{|c|}{ Application } \\
\hline & vision & $\begin{array}{c}\text { circadian - day shift } \\
\text { work }\end{array}$ & $\begin{array}{l}\text { circadian - night } \\
\text { shift work }\end{array}$ \\
\hline quantity & $\begin{array}{c}\text { low } \\
\text { (300-500 lux on task } \\
\sim 100 \text { lux at eye) }\end{array}$ & $\begin{array}{c}\text { high } \\
(\sim 1000 \text { lux at eye })\end{array}$ & $\begin{array}{c}\text { high } \\
(\sim 1000 \text { lux at eye })\end{array}$ \\
\hline spectrum & $\begin{array}{c}\text { photopic (peak sensitivity } \\
555 \mathrm{~nm} \text { ) }\end{array}$ & $\begin{array}{c}\text { short-wavelength } \\
\text { (peak sensitivity } \\
420-480 \mathrm{~nm} \text { ) }\end{array}$ & $\begin{array}{c}\text { short-wavelength } \\
\text { (peak sensitivity } \\
420-480 \mathrm{~nm} \text { ) }\end{array}$ \\
\hline $\begin{array}{c}\text { spatial } \\
\text { distribution }\end{array}$ & $\begin{array}{c}\text { distribution important } \\
\text { (task luminance, contrast } \\
\text { and size determine } \\
\text { visibility) }\end{array}$ & $\begin{array}{l}\text { independent of } \\
\text { distribution } \\
\text { (illuminance at eye) }\end{array}$ & $\begin{array}{c}\text { independent of } \\
\text { distribution } \\
\text { (illuminance at eye) }\end{array}$ \\
\hline timing & any time & subjective morning & $\begin{array}{c}\text { periodically } \\
\text { throughout shift }\end{array}$ \\
\hline duration & very short (less than $1 \mathrm{~s}$ ) & long (1-2 hr) & $\begin{array}{l}\text { short }(15 \mathrm{~min} \\
\text { pulses }\end{array}$ \\
\hline
\end{tabular}

Table 1. A proposed framework for lighting practice considering circadian photobiology (Rea et al., 2002[20]). 


\section{Quantity}

Most visual processing can be adequately performed under quite low levels of light[22], as evidenced by the lighting recommendations for exterior locations published by the Illuminating Engineering Society of North America[14]. When we consider that outdoor daylight levels regularly exceed 10,000 lux, and that this cycle has been responsible for maintaining circadian entrainment for millions of years, it should not be surprising that the human circadian system requires significantly higher quantities of light to maintain entrainment or shift circadian rhythms[12, 15-18], compared to what the visual system needs to function. In comparison, indoor light levels - except close to windows - are relatively low[20]; while these are more than sufficient for visual performance, they often fall below the threshold for influencing the human circadian system (see Figure 1).

\section{Spectrum}

At typical indoor light levels, the visual system is driven almost exclusively by cone photoreceptors, which are most sensitive to the middle-wavelength (green-yellow) portion of the visible spectrum. At levels typical of many outdoor applications (such as parking areas and roads), the spectral sensitivity of the peripheral

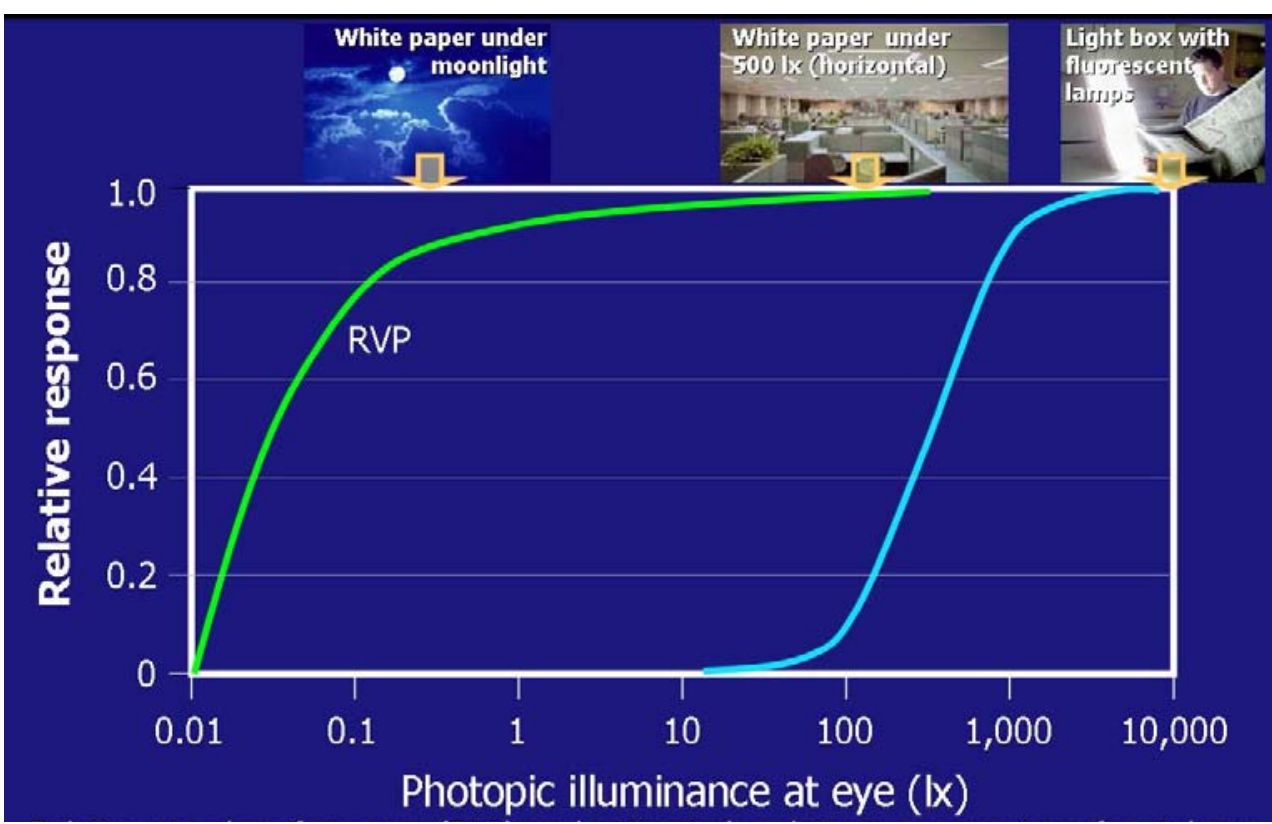

Figure 1. Relative visual performance (RVP) and estimated melatonin suppression after 1 hour exposure to fluorescent illumination (based on McIntire, et al., 1989)[10].

visual system shifts

toward shorter wavelength (blue-green) light $[23,24]$, representing a mixture of cone and rod photoreceptor inputs at these levels.

The human circadian system, in comparison, is most sensitive to the short-wavelength (blue) portion of the visible spectrum[17, 18, 25-30]. In simple language, the human circadian system is a "blue sky detector." A combination of known photoreceptors (rods and cones) with a recently discovered novel photoreceptor in the retina[31-33] known as intrinsically-photoreceptive retinal ganglion cells — which are maximally sensitive to blue light — participate in circadian phototransduction. For the same illuminance measured at the eye, an hour-long exposure from $7500 \mathrm{~K}$ fluorescent lamps ("cool" in appearance) is about twice as effective at suppressing nocturnal melatonin than exposure to the same measured light level and duration from $3000 \mathrm{~K}$ 
fluorescent lamps ("warm" in appearance)[20]. In terms of illumination for visual performance, they would be equivalent[22].

It has recently been shown that the human circadian system seems to respond to light in such a way that adding long-wavelength (yellow) light to short-wavelength (blue) light actually reduces its effectiveness[34,35]. This could help partially explain why very high quantities of white light - containing light in the blue and yellow spectral regions - are required for circadian regulation, while just a few lux of short-wavelength (blue) light are sufficient for obtaining these responses $[25,26,29,30,34,35]$.

\section{Distribution}

Patterns of light and dark are crucial for the visual system. Indeed, without luminance contrast, most of what we see would be invisible. The circadian system, in comparison, appears to be concerned primarily with the total amount of light reaching the retina. Recently, some evidence has been published that light from above the horizon is more effective than light from below the horizon in suppressing melatonin[36]. This makes sense, given the fact that the sky is the primary light source to which the circadian system has been tuned.

\section{Timing}

The visual system can respond to light stimulus at any time of day. The timing of exposure, however, can result in either phase advances, phase delays, or no effect on the circadian clock[37]. As discussed above, advances shift the master clock earlier, while delays shift it later, so that light applied during the morning will cause a phase advance, while light applied in the evening will cause a phase delay.

\section{Duration}

The visual system can respond to light stimuli in less than a second[38]. The duration of light exposure needed to suppress melatonin in humans, on the other hand, is much longer. Studies from the literature $[12,15,16]$ show that suppression of melatonin content in the bloodstream in humans starts approximately 10 minutes after the initiation of bright light exposure.

\section{Interactions}

The characteristics of light discussed here and summarized in Table 1 can interact with one another. Reiterating the discussion of spectral sensitivity, a relatively low level of blue light can as effectively influence the human circadian system as a very high level of white light. Additionally, evidence that even modest levels of white light, when presented for very long durations[39] can impact melatonin production, is surfacing. And again, without considering the time of day that lighting is applied, no amount of light—regardless of its intensity — can be guaranteed to impact on the circadian system. Finally, the photic history, i.e., the amount of light one is exposed to during the day, will influence the sensitivity to light at night for melatonin suppression[40]. 


\section{Lighting and Alertness}

It is becoming increasingly clear that relatively short exposures to light can positively impact alertness, wakefulness, and even performance of some tasks, if only temporarily. Badia et al.[41] studied the effects of bright white light on body temperature, alertness, and performance. Subjects were exposed to 90-minute periods of alternating bright (5000 to 10,000 lux) and dim (50 lux) light during daytime and nighttime. Body temperature, alertness (measured via electrophysiological brain activity) and performance were higher following bright light exposure, compared to after dim light exposure during nighttime hours, but not this effect was not seen during the daytime. Campbell et al.[42] exposed subjects to bright (1000 lux) and dim (10 and 100 lux) light levels for 8 hours at night. Subjective and objective measures showed that subjects were more alert after bright light exposure; dim light exposure again failed to influence these measures.

Boyce et al.[43] exposed subjects working in a night shift routine over three nights to four different lighting conditions (using white light): low (250 lux), high (2800 lux), increasing (from 200 to 2800 lux over the shift), and decreasing (from 2800 to 200 lux over the shift). They found that the two conditions using early bright light (the high and decreasing conditions) improved performance of some tasks, increased arousal level, improved sleep quality throughout the experiment, and delayed the time that subjects went to bed after completing the night shift. Figueiro et al.[19] exposed night-shift nurses to 15 minutes of bright white (at least 500 lux at the eye) or to dim (less than 100 lux) light. They found that feelings of wakefulness, alertness, and well-being were improved after the bright light, relative to the dim light condition.

Cajochen et al.[44] exposed subjects to light levels ranging from 3 to 9100 lux for 6.5 hours during the night. They found a short-term alerting effect of the light, using recorded electrophysiological brain activity, and also found a reduction in feelings of sleepiness. Building on the evidence that light can have alerting effects at night and on the knowledge that the circadian system is maximally sensitive to short-wavelength (blue light) radiation, Cajochen et al.[45] reported results similar to Badia et al.[41], but used only 5 lux of blue light for a about 40 minutes, in comparison with the much higher light levels and longer durations of white light used by Badia et al.[41].

\section{Implications for the Submarine Environment}

Research on the potential alerting effects of light has not been performed using Submariners as subjects, but the impacts on this population should not differ greatly. These individuals often need to make complex decisions around the clock. The evidence summarized above suggests that using light to influence circadian rhythms and increasing alertness could play a crucial role in increasing safety and crew effectiveness on submarines.

Specifically, the issues discussed above have several potential implications:

- $\quad$ Large quantities of light, which could contribute to discomfort glare[46] and reduce visibility of critical displays, might not be needed if spectrally-tuned lighting can be incorporated into the submarine environment. 
- $\quad$ Because the location of light sources for circadian regulation is relatively unimportant (compared to quantity and spectral distribution, for example), new approaches to delivering low levels of blue light (such as via display screens, or in rest areas) could perhaps be explored.

- $\quad$ Near-term impacts of light on alertness might be able to last several hours under some conditions[43]. For this reason, incorporating lighting systems for this purpose in break and rest areas, in the form of "light showers" might be feasible.

- $\quad$ Because the human circadian system responds maximally to short-wavelength (blue) light, the use of yellow-tinted glasses - when color recognition is not a crucial visual task - could provide "circadian darkness" that might allow personnel to temporarily remain active while avoiding potentially disruptive impacts of light on sleep patterns.

No doubt, much further research will be necessary before circadian criteria can be considered to have equal weight as visual criteria in the design of lighting in environments such as submarines, but it is becoming increasingly clear that such considerations will eventually need to be made. We hope this short paper can stimulate discussion among researchers and decision-makers alike to assess how - and when — such a paradigm shift could occur. 


\section{REFERENCES}

1. Stolgitis, W., Effects of sleep loss and demanding work/rest cycles: An analysis of the traditional Navy watch system and a proposed alternative. 1969, Navy Postgraduate School: Monterey. p. 28.

2. Withrow, R.B., ed. Photoperiodism. 1959, American Association for the Advancement of Science: Washington, D.C.

3. Halberg, F., et al., Physiologic 24-Hour Periodicity in Human Beings and Mice: The Lighting Regimen and Daily Routine, in Photoperiodism, R. Withrow, Editor. 1959, American Association for the Advancement of Science: Washington, D.C.

4. Moore-Ede, M.C., F.M. Sulzmann, and C.A. Fuller, The Clocks That Time Us. 1982, Cambridge: Harvard University Press.

5. Shibui, K., et al., Periodic fatigue symptoms due to desynchronization in a patient with non-24-h sleep-wake syndrome. Psychiatry Clin Neurosci, 1998. 52(5): p. 477-81.

6. Gupta, S. and A.K. Pati, Desynchronization of circadian rhythms in a group of shift working nurses: effects of pattern of shift rotation. J Hum Ergol (Tokyo), 1994. 23(2): p. 121-31.

7. Reinberg, A., et al., Internal desynchronization of circadian rhythms and tolerance of shift work. Chronobiologia, 1989. 16(1): p. 21-34.

8. Boggild, H. and A. Knutsson, Shift work, risk factors and cardiovascular disease. Scand J Work Environ Health, 1999. 25(2): p. 85-99.

9. Scott, A.J., Shift work and health. Prim Care, 2000. 27(4): p. 1057-79.

10. Karlsson, B., A. Knutsson, and B. Lindahl, Is there an association between shift work and having a metabolic syndrome? Results from a population based study of 27,485 people. Occup Environ Med, 2001. 58(11): p. 747-52.

11. Akerstedt, T., Sleepiness as a consequence of shift work. Sleep, 1988. 11(1): p. 17-34.

12. Lewy, A.J., et al., Light suppresses melatonin secretion in humans. Science, 1980. 210(4475): p. 1267-9.

13. Kelly, T.L., et al., Nonentrained circadian rhythms of melatonin in submariners scheduled to an 18-hour day. J Biol Rhythms, 1999. 14(3): p. 190-6.

14. Rea, M.S., ed. IESNA Lighting Handbook: Reference and Application. Vol. 9. 2000, Illuminating Engineering Society of North America: New York.

15. McIntyre, I.M., et al., Human melatonin suppression by light is intensity dependent. J Pineal Res, 1989. 6(2): p. 149-56.

16. McIntyre, I.M., et al., Quantal melatonin suppression by exposure to low intensity light in man. Life Sci, 1989. 45(4): p. 327-32.

17. Rea, M.S., J.D. Bullough, and M.G. Figueiro, Human melatonin suppression by light: $a$ case for scotopic efficiency. Neurosci Lett, 2001. 299(1-2): p. 45-8.

18. Rea, M.S., J.D. Bullough, and M.G. Figueiro, Phototransduction for human melatonin suppression. J Pineal Res, 2002. 32(4): p. 209-13.

19. Figueiro, M.G., et al., The Effects of Bright Light on Day and Night Shift Nurses' Performance and Well-Being in the NICU. Neonatal Intensive Care, 2001. 14: p. 29-32.

20. Rea, M.S., M.G. Figueiro, and J.D. Bullough, Circadian Photobiology: An Emerging Framework for Lighting Practice and Research. Lighting Research and Technology, 2002. 34: p. 177-190. 
21. Rea, M.S., et al., A model of phototransduction by the human circadian system. Brain Res Brain Res Rev, 2005. 50(2): p. 213-28.

22. Rea, M.S. and M.J. Ouellette, Relative Visual Performance: A Basis for Application. Lighting Research and Technology, 1991. 23: p. 135-144.

23. He, Y., et al., Evaluating Light Source Efficacy Under Mesopic Conditions Using Reaction Times. Journal of the Illuminating Engineering Society, 1997. 26: p. 125-138.

24. Rea, M.S., et al., A Proposed Unified System of Photometry. Lighting Research and Technology, 2004. 36: p. 85-111.

25. Brainard, G.C., et al., Action spectrum for melatonin regulation in humans: evidence for a novel circadian photoreceptor. J Neurosci, 2001. 21(16): p. 6405-12.

26. Thapan, K., J. Arendt, and D.J. Skene, An action spectrum for melatonin suppression: evidence for a novel non-rod, non-cone photoreceptor system in humans. J Physiol, 2001. 535(Pt 1): p. 261-7.

27. Wright, H.R. and L.C. Lack, Effect of Light Wavelength on Suppression and Phase Delay of the Melatonin Rhythm. Chronobiology International, 2001. 18: p. 801-808.

28. Wright, H.R., L.C. Lack, and D.J. Kennaway, Differential Effects of Light Wavelength in Phase Advancing the Melatonin Rhythm. Journal of Pineal Research, 2004. 36: p. 140144.

29. Lockley, S.W., G.C. Brainard, and C.A. Czeisler, High sensitivity of the human circadian melatonin rhythm to resetting by short wavelength light. J Clin Endocrinol Metab, 2003. 88(9): p. 4502-5.

30. Warman, V.L., et al., Phase advancing human circadian rhythms with short wavelength light. Neurosci Lett, 2003. 342(1-2): p. 37-40.

31. Berson, D.M., F.A. Dunn, and M. Takao, Phototransduction by retinal ganglion cells that set the circadian clock. Science, 2002. 295(5557): p. 1070-3.

32. Dacey, D.M., et al., Melanopsin-expressing ganglion cells in primate retina signal colour and irradiance and project to the LGN. Nature, 2005. 433(7027): p. 749-54.

33. Hattar, S., et al., Melanopsin-containing retinal ganglion cells: architecture, projections, and intrinsic photosensitivity. Science, 2002. 295(5557): p. 1065-70.

34. Figueiro, M.G., et al., Demonstration of additivity failure in human circadian phototransduction. Neuro Endocrinol Lett, 2005. 26(5): p. 493-8.

35. Figueiro, M.G., et al., Preliminary evidence for spectral opponency in the suppression of melatonin by light in humans. Neuroreport, 2004. 15(2): p. 313-6.

36. Glickman, G., et al., Inferior retinal light exposure is more effective than superior retinal exposure in suppressing melatonin in humans. J Biol Rhythms, 2003. 18(1): p. 71-9.

37. Jewett, M.E., et al., Human circadian pacemaker is sensitive to light throughout subjective day without evidence of transients. Am J Physiol, 1997. 273(5 Pt 2): p. R18001809.

38. Ingling, C.R., Jr., E. Martinez, and A.L. Lewis, Tonic-phasic-channel dichotomy and Crozier's law. J Opt Soc Am, 1983. 73(2): p. 183-9.

39. Zeitzer, J.M., et al., Sensitivity of the human circadian pacemaker to nocturnal light: melatonin phase resetting and suppression. J Physiol, 2000. 526 Pt 3: p. 695-702.

40. Hebert, M., et al., The effects of prior light history on the suppression of melatonin by light in humans. J Pineal Res, 2002. 33(4): p. 198-203.

41. Badia, P., et al., Bright light effects on body temperature, alertness, EEG and behavior. Physiol Behav, 1991. 50(3): p. 583-8. 
42. Campbell, S.S. and D. Dawson, Enhancement of nighttime alertness and performance with bright ambient light. Physiol Behav, 1990. 48(2): p. 317-20.

43. Boyce, P.R., et al., Lighting the Graveyard Shift: The Influence of a Daylight-Simulating Skylight on the Task Performance and Mood of Nightshift Workers. Lighting Research and Technology, 1997. 29: p. 105-134.

44. Cajochen, C., et al., Dose-response relationship for light intensity and ocular and electroencephalographic correlates of human alertness. Behav Brain Res, 2000. 115(1): p. 75-83.

45. Cajochen, C., et al., High sensitivity of human melatonin, alertness, thermoregulation, and heart rate to short wavelength light. J Clin Endocrinol Metab, 2005. 90(3): p. 13111316.

46. Fry, G.A., Evaluating Disability Effects of Approaching Automobile Headlights, H.R.B. 89, Editor. 1954, National Research Council. p. 38-42.

Presented at the 2006 Undersea Human Systems Integration Symposium, Mystic, CT, June 6-8. Newport, RI: Naval Undersea Warfare Center. 